\title{
MR OF AN ADRENAL PSEUDOCYST
}

\author{
Alex M. Aisen,* Dana A. Ohl, $\nmid$ Thomas L. Chenevert, ${ }^{*}$ \\ Philip Perkins, $\ddagger$ AND Wesley MiKesell \\ *Department of Radiology, †Division of Urology, Department of Surgery, and \\ ¥Department of Pathology, University of Michigan Hospitals, Ann Arbor, MI 48109-0030, USA
}

\begin{abstract}
We describe the appearance of an adrenal pseudocyst on MRI and CT. The MR characteristics of the lesion were noteworthy in that the lesion had two components with different imaging characteristics. The larger component was of low signal intensity on both $T_{1}$-and $T_{2}$-weighted images and might have been confused with an adrenal adenoma.
\end{abstract}

Keywords: Magnetic resonance imaging; Adrenal pseudocysts.

Adrenal pseudocysts are uncommon lesions of the adrenal which may derive from metamorphosis of endothelial adrenal cysts or occur as a consequence of prior adrenal hemorrhage. ${ }^{1,2}$ We report a case of this entity which had unusual imaging characteristics on both CT and MRI. In particular, the bulk of the lesion had MR signal intensity properties superficially suggestive of an adenoma.

\section{CASE REPORT}

A 68-yr-old male physician with a history of gout presented with hematuria. One year earlier he was in a motor vehicle accident and sustained several broken ribs. An excretory urogram performed at an outside institution revealed a left suprarenal mass (reportedly not clearly separable from the kidney). A CT scan, also performed at an outside institution, revealed a well-defined $5 \mathrm{~cm}$ left adrenal mass (Fig. 1). The bulk of the tumor was low density with a high density central portion. Punctate calcifications were present. The patient was referred to this institution and an MR scan was obtained on a $0.35 \mathrm{~T}$ Toshiba (formerly Diasonics) instrument (Fig. 2). The lesion had low signal intensity on the $T_{1}$-weighted images. On the $T_{2}$-weighted scans most of the lesion had a low signal intensity; the central region corresponding to the dense area on CT had high signal. Urinary catecholamines were normal.
Minimally elevated 17 -ketosteroid levels prompted an NP-59 study which revealed normal uptake in the right adrenal gland $i$ nd absent tracer accumulation in the left.

Pertinent quantitative MR measurements were as follows. On the $T_{2}$-weighted image (TR $=2000 \mathrm{msec}$, $\mathrm{TE}=60 \mathrm{msec}$ ), the intensity ratio of the peripheral portion of the lesion to fat was 0.73 , to liver was 1.13 , and to muscle was 3.17. For the central high intensity zone, the ratios were $1.08,1.66$, and 4.69 , respectively. The $T_{2}$ times of the peripheral and central portions of the lesion, estimated from the signal intensities on the first and second echo images, were 100 and $144 \mathrm{msec}$, respectively. These $T_{2}$ values must be interpreted with considerable caution, since measurements made from double ccho abdominal images are subject to substantial measurement error.* ${ }^{*}$ The $T_{1}$ times were measured

*The measurements are included because such values are commonly reported in the literature. Repeat measurements using redrawn regions-of-interest on the same slice yielded similar values; however, regions-of-interest drawn on an adjacent slice yielded substantially different values: $81 \mathrm{msec}$ for the central portion and $55 \mathrm{msec}$ for the peripheral zone. This variability reflects both the heterogencity of this particular lesion and the errors inherent in MRI estimates of $T_{2}$ made from image pairs. The intensity ratios of lesion to normal tissues were more robust, in that they were more similar on the adjacent slices.
ReCEIVED 2/19/92; ACCEPTED 4/14/92.

Address correspondence to Alex M. Aisen, M.D., Depart- ment of Radiology, University of Michigan Hospitals, 1500 E. Medical Center Drive, Ann Arbor, MI 48109-0030, USA. 


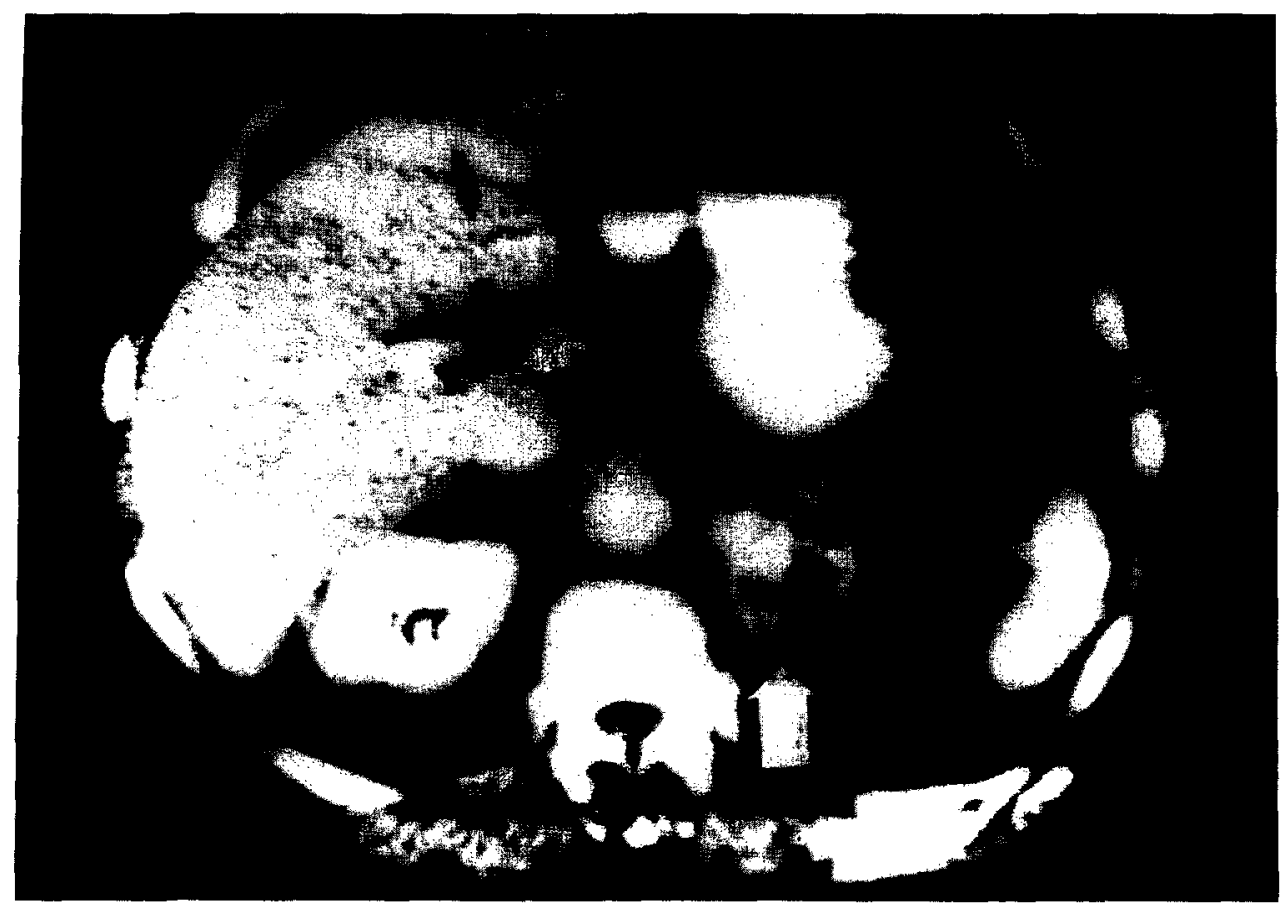

Fig. 1. CT scan reveals a $5 \mathrm{~cm}$ left adrenal mass with a relatively low density peripheral component and a smaller, higher density central region.

ex vivo from samples cut from the surgical specimen on an $0.5 \mathrm{~T}$ IBM Minispec spectrometer at $40^{\circ} \mathrm{C}$, and were $637 \mathrm{msec}$ and $612 \mathrm{msec}$, respectively.

The imaging and biochemical studies did not provide a definitive diagnosis. Consideration was given to an adrenal adenoma with central hemorrhage or, conceivably, malignant degeneration. Because of the large size of the lesion, surgical excision was performed. Pathology revealed an adrenal pseudocyst consisting primarily of fibrin (Fig. 3). There were some areas of old hemorrhage, but a well-defined area corresponding to the central portion seen on MR and CT could not be prospectively identified.

\section{DISCUSSION}

Magnetic resonance imaging is now well known to be of use in the characterization of adrenal lesions. ${ }^{3-11}$ On $T_{2}$-weighted MR images, particularly those acquired with lower or intermediate field strength scanners, ${ }^{6}$ benign adrenal adenomas generally have low signal intensity while most other lesions, including adrenal cortical carcinoma, pheochromocytoma, and metastatic deposits have high signal.

In contrast to this case, most adrenal pseudocysts have been previously reported to be of water density and homogeneous on CT. ${ }^{12}$ The MR appearance of this lesion was also unusual. The lesion was dark on the $T_{1}$-weighted images; however, it had variable signal characteristics on the $T_{2}$-weighted images with a central high signal area and a larger, low density peripheral portion.

The low signal component might have been mistaken for an adenoma, possibly with central hemorrhage or, conceivably, with malignant degeneration. The perceived low signal intensity on the $T_{2}$-weighted image compared to liver is consistent with an adenoma ${ }^{3,3,6,8-11}$ and unusual for other common adrenal lesions. ${ }^{3,5,6,8-10}$ Though benign adenomas may occasionally have high signal intensities, ${ }^{4,11,13}$ very few nonadenoma lesions of the adrenal have been previously described with low signal intensity ${ }^{4-6,8-10}$; however, the high calculated $T_{2}$ time would be very unusual for an adenoma ${ }^{3,5,6}$ and suggests a different diagnosis.

Previously described adenomas usually have lower estimated $T_{2}$ times. The high relaxation time may be a consequence of relatively acellular "fluid-like" properties of the fibrinous pseudocyst. The reduced signal of the peripheral component is presumably a consequence of the long $T_{1}$ time and possibly a low mobile proton content (the latter was not measured). Hemo- 


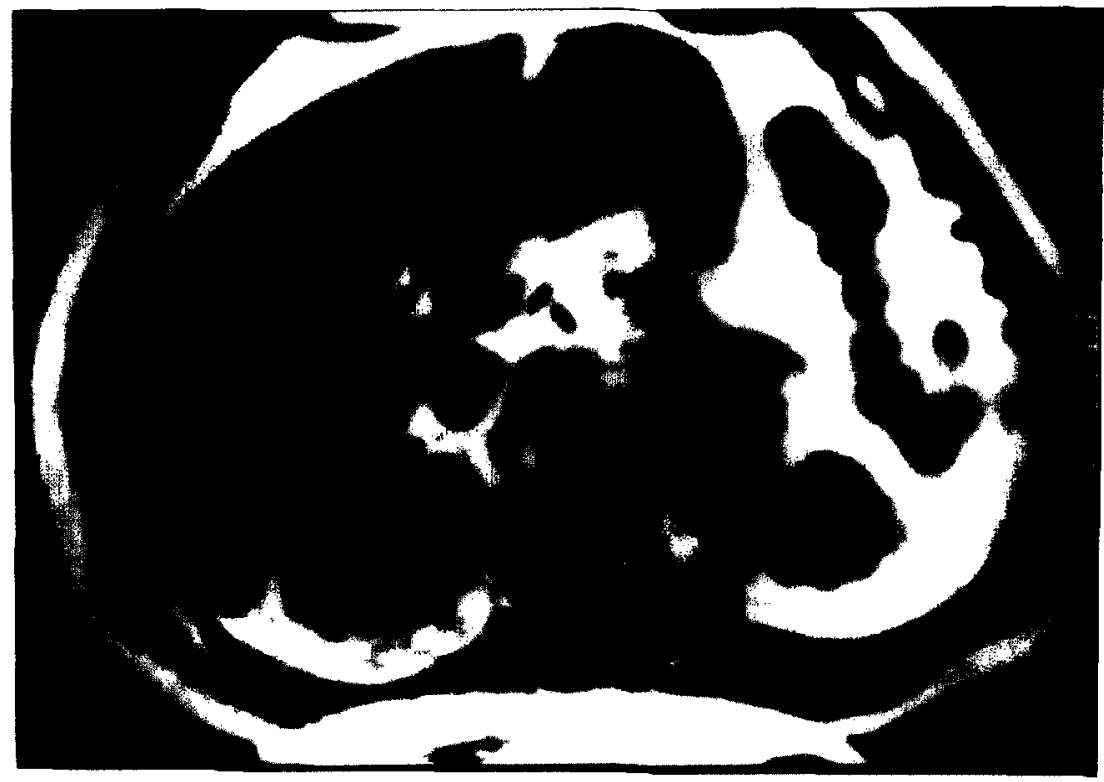

(A)

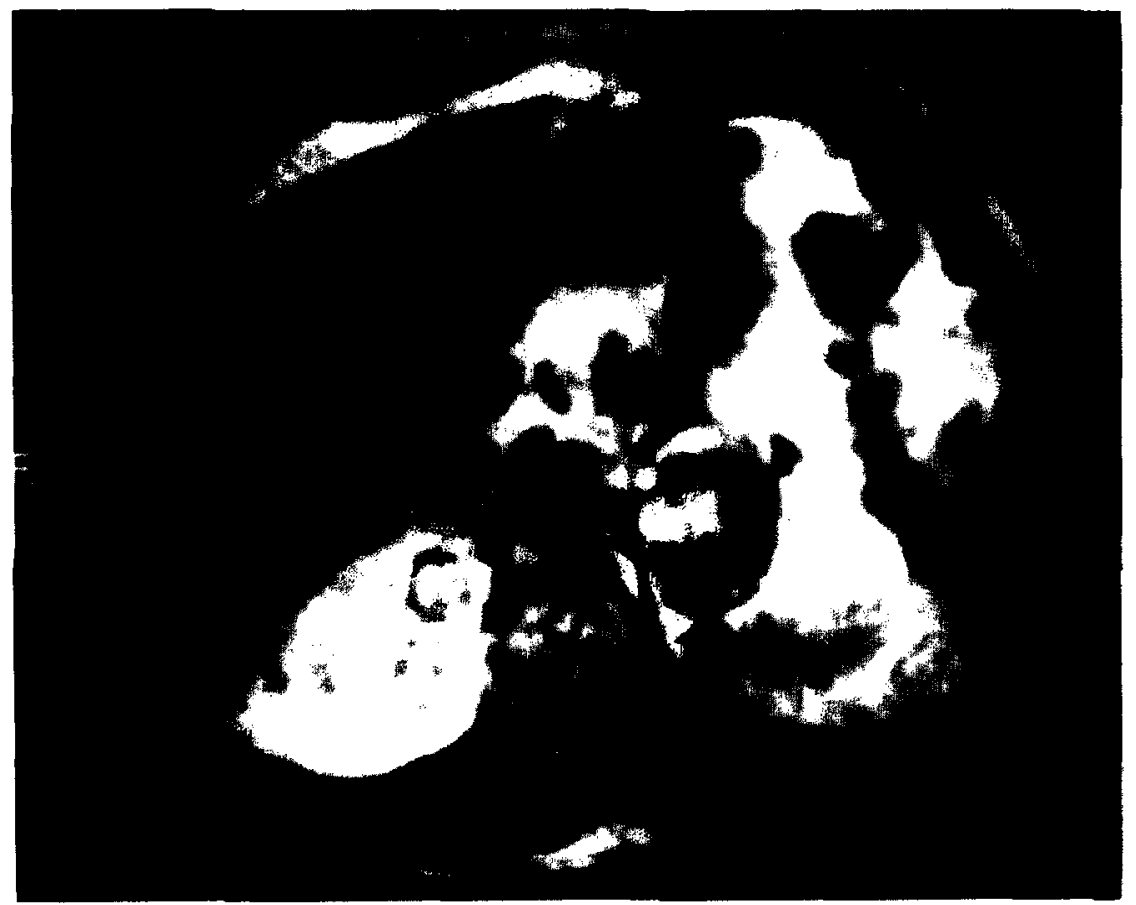

(B)

Fig. 2. MRI demonstrates a uniformly hypointense lesion on (A) $T_{1}$-weighted images (TR/TE $=300 / 15$ ). With (B) $T_{2}$ weighting $(T R / T E=2000 / 60)$, the lesion displays a low intensity peripheral zone and a high intensity central zone, matching the CT image.

siderin deposition can result in diminished signal intensity, but this does not seem to be the case here since the estimated $T_{2}$ times were relatively long. The central area of high signal on the $T_{2}$-weighted image and in- creased density on CT are not fully explained by the available pathologic data.

In addition to illustrating the appearance of one instance of an adrenal pseudocyst, this case confirms 


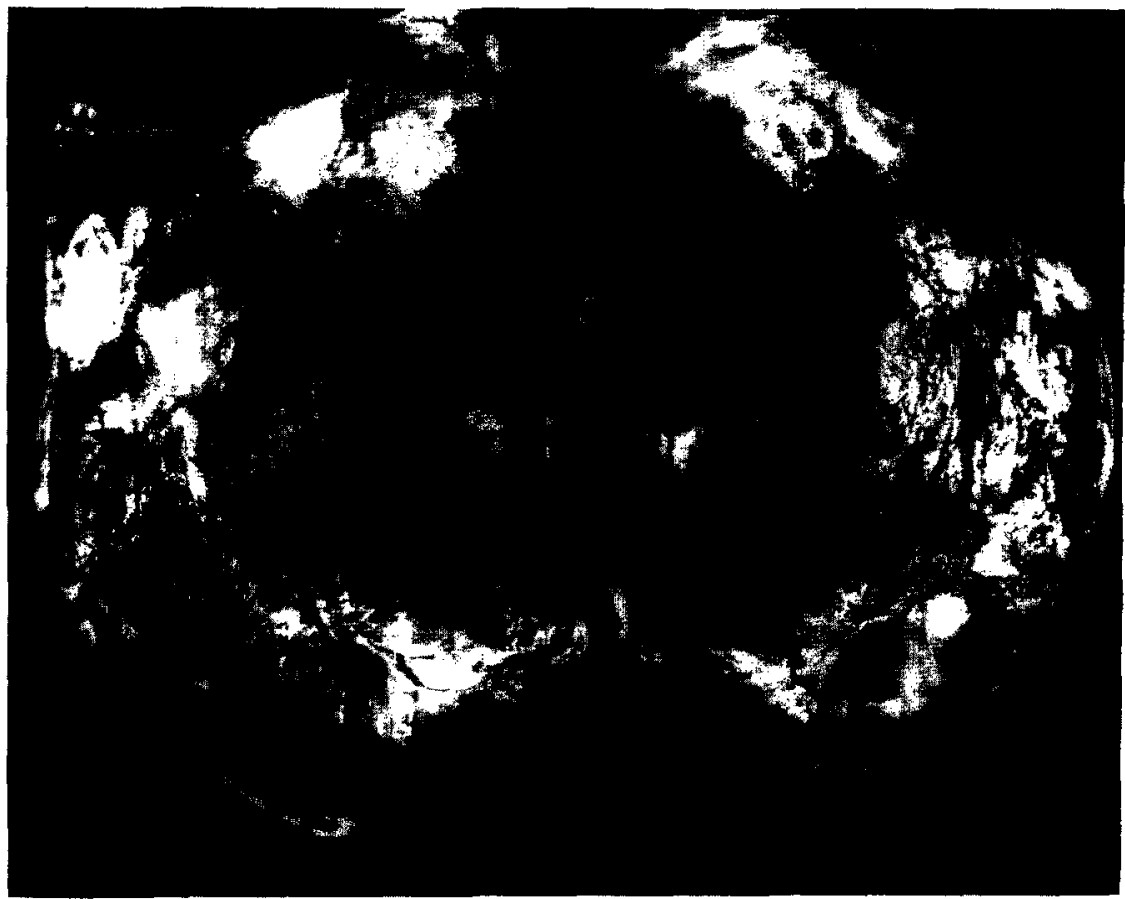

Fig. 3. Cross section of the specimen. No clear distinction between the central and peripheral zones was evident. Remaining normal adrenal gland is evident (arrow).

that care is required in interpreting signal intensity ratios.

\section{REFERENCES}

1. Cotran, R.S.; Robbins, S.L.; Kumar, V. Robbins' Pathologic Basis of Disease. 4th ed. Philadelphia: W.B. Saunders; 1989.

2. Gaffey, M.J.; Mills, S.E.; Fechner, R.E.; Bertholf, M.F.; Allen, M.S., Jr. Vascular adrenal cysts: A clinicopathologic and immunohistochemical study of endothelial and hemorrhagic (pseudocystic) variants. $A m$. $J$. Surg. Pathol. 13:740-747; 1989.

3. Baker, M.E.; Blinder, R.; Spritzer, C.; Leight, G.S.; Herfkens, R.J.; Dunnick, N.R. MR evaluation of adrenal masses at 1.5 T. Am. J. Roentgenol. 153:307-312; 1989.

4. Chang, A.; Glazer, H.S.; Lee, J.K.; Ling, D.; Heiken, J.P. Adrenal gland: MR imaging. Radiology 163:123$128 ; 1987$.

5. Glazer, G.M.; Woolsey, E.J.; Borrello, J.; Francis, I.R.; Aisen, A.M.; Bookstein, F.; Amendola, M.A.; Gross, M.D.; Bree, R.L.; Martel, W. Adrenal tissue characterization using MR imaging. Radiology 158:73-79; 1986.

6. Kier, R.; McCarthy, S. MR characterizution of adrenal masses: Field strength and pulse sequence considerations. Radiology 171:671-674; 1989.
7. Leroy-Willig, A.; Bittoun, J.; Luton, J.P.; Louvel, A.; Lefevre, J.E.; Bonnin, A.; Roucayrol, J.C. In vivo MR spectroscopic imaging of the adrenal glands: Distinction between adenomas and carcinomas larger than $15 \mathrm{~mm}$ based on lipid content. Am. J. Roentgenol. 153:771$773 ; 1989$.

8. Reinig, J.W.; Doppman, J.l.; Dwyer, A.J.; Frank, J. MRI of indeterminate adrenal masses. Am. J. Roentgenol. 147:493-496; 1986.

9. Reinig, J.W.; Doppman, J.L.; Dwyer, A.J.; Johnson, A.R.; Knop, R.H. Distinction between adrenal adenomas and metastases using MR imaging. J. Comput. Assist. Tomogr. 9:898-901; 1985.

10. Reinig, J.W.; Doppman, J.L.; Dwyer, A.J.; Johnson, A.R.; Knop, R.H. Adrenal masses differentiated by MR. Radiology 158:81-84; 1986.

11. Remer, E.M.; Weinfeld, R.M.; Glazer, G.M.; Quint, L.E.; Francis, 1.R.; Gross, M.D.; Bookstein, F.L. Hyperfunctioning and nonhyperfunctioning benign adrenal cortical lesions: Characterization and comparison with MR imaging. Radiology 171:681-685; 1989.

12. Johnson, C.D.; Baker, M.E.; Dunnick, N.R. CT demonstration of an adrenal pseudocyst. J. Comput. Assist. Tomogr. 9:817-819; 1985.

13. Baker, M.E.; Spritzer, C.; Blinder, R.; Herfkens, R.J.; Leight, G.S.; Dunnick, N.R. Benign adrenal lesions mimicking malignancy on MR imaging. Report of two cases. Radiology 163:669-671; 1987. 